\title{
Nonlinear Decoupled Motion-Stiffness Control and Collision Detection/Reaction for the VSA-II Variable Stiffness Device
}

\author{
Alessandro De Luca Fabrizio Flacco \\ Dipartimento di Informatica e Sistemistica \\ Università di Roma "La Sapienza" \\ Via Ariosto 25, 00185 Roma, Italy \\ \{deluca,fflacco\}@dis.uniroma1.it
}

\author{
Antonio Bicchi Riccardo Schiavi \\ Centro "E. Piaggio" \\ Università di Pisa \\ Via Diotisalvi 2, 56100 Pisa, Italy \\ \{bicchi,riccardo.schiavi\}@ing.unipi.it
}

\begin{abstract}
Variable Stiffness Actuation (VSA) devices are being used to jointly address the issues of safety and performance in physical human-robot interaction. With reference to the VSA-II prototype, we present a feedback linearization approach that allows the simultaneous decoupling and accurate tracking of motion and stiffness reference profiles. The operative condition that avoids control singularities is characterized. Moreover, a momentum-based collision detection scheme is introduced, which does not require joint torque sensing nor information on the time-varying stiffness of the device. Based on the residual signal, a collision reaction strategy is presented that takes advantage of the proposed nonlinear control to rapidly let the arm bounce away after detecting the impact, while limiting contact forces through a sudden reduction of the stiffness. Simulations results are reported to illustrate the performance and robustness of the overall approach. Extensions to the multidof case of robot manipulators equipped with VSA-II devices are also considered.
\end{abstract}

\section{INTRODUCTION}

A successful paradigm in physical Human-Robot Interaction (pHRI) is to design robots for safety and to control them for performance [1], [2]. In the recent years, this codesign has led to the development of several manipulators and actuation systems that integrate in different ways the common concepts of lightweight link structure [3], compliant transmissions and arm coverage [4], [5], hybrid actuation with remote displacement of the main motors [6], and variable stiffness/impedance actuators (VSA/VIA). In particular, VSA devices may either have a passive variation of joint stiffness [7], or actively modify it, with an antagonistic arrangement of two motors [8]-[10] or with separate actuation for motion and stiffness [11], [12]. All the above mechani$\mathrm{cal} / \mathrm{actuation}$ choices allow to reduce intrinsically the risk of user injuries resulting from possible unexpected collisions of a robot that closely cooperates with humans [13], [14].

From the point of view of performance, control design should be aimed at compensating for the static deflection and dynamic vibrations associated to the presence of (constant or time-varying) compliant transmissions, so as to accurately execute fast motions as in the rigid case. For instance, model-based nonlinear feedback control allows to cancel completely these effects in robots with elastic joints of constant stiffness [15], [16]. For some instances of 1-dof arms with variable stiffness actuation, feedback linearization has been proven effective in controlling both the link motion and the desired stiffness in an antagonistic case [17] and in one with separate actuation [11]. More in general, two classes of multi-dof robots with variable joint stiffness have been considered in [18], one where the stiffness can be modified instantaneously by a specific input command and another where this additional control input modulates the stiffness through a second-order mechanical system. Depending on the class type, a dynamic or, respectively, a static feedback linearization law was shown to be sufficient for the simultaneous and decoupled control of the motion and stiffness outputs. Nonetheless, the feasibility of these general control approaches depends on the chosen mechanical implementation of variable stiffness actuation. As a matter of fact, one should check the invertibility of the so-called decoupling matrix of the system on a case-by-case basis.

A second fundamental task for the robot controller is the handling of collisions, which include their fast and reliable detection and the switching to a safe reaction strategy once a collision has been detected. Human-robot collisions may happen in general at any location along the robot structure. While external vision systems or proximity sensing and additional force/torque sensors are indeed helpful for the anticipation or avoidance of collisions, a simpler and more cost-effective detection approach should rely only on the available proprioceptive sensors of the robot (encoders) and on the use of its nominal dynamic model. For rigid robots, such collision detectors have been designed, e.g., in [19], [20]. In particular, the momentum-based approach proposed in [20] regards the collision as a system fault [21] and generates a residual vector that can be used both for detection and for the successive reaction. Moreover, this detection scheme can be easily extended to the case of robots with joint elasticity, provided that joint torque sensors are available [22]. No such results are available for detecting collisions in the case of variable stiffness actuation.

Upon recognition of a collision, the simplest reaction strategy is to stop the robot [23], [24], which is safe but leaves the human user in an unpleasant state of danger, next to the robot arm. Canceling gravity through control [25] allows to let the robot float in space, reacting in response to the impact forces in an unbiased way. More active strategies, where the residual vector is used to define a direction of safe reflex motion of the robot, have been proposed and 


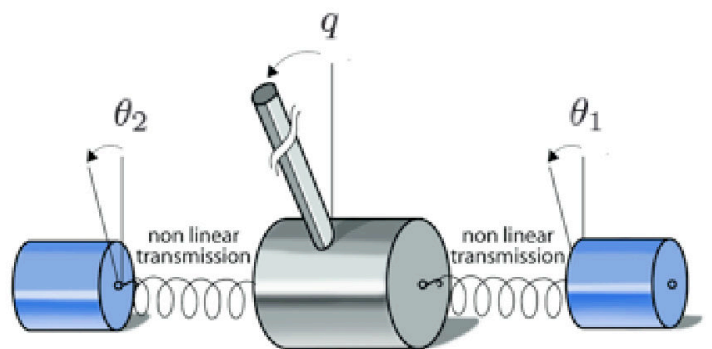

Fig. 1. VSA-II antagonistic principle (modified from [10])

successfully experimented in [14], [22], both in the case of rigid and of elastic joints. The definition of effective strategies of reaction to collisions for manipulators with variable stiffness actuation is still an open research issue.

In this paper, we present a complete approach to the accurate and safe control of a 1-dof arm equipped with the latest VSA-II prototype developed at the University of Pisa [10]. After recalling the dynamic model of the VSAII in Sect. II, the control design for tracking smooth, but otherwise arbitrary, reference trajectories for arm position and stiffness is presented in Sect. III. In particular, we characterize the singularities of the feedback linearization law for the VSA-II, and show how these can be avoided by suitably pre-loading the device. Section IV introduces an efficient scheme for detecting the occurrence of unexpected collisions without measuring transmission torques at the joint. An active reaction strategy is proposed, taking advantage of the feedback linearizing controller. Section $\mathrm{V}$ reports illustrative simulation results for free motion and collision, both in the nominal case and under model perturbations. The paper is mainly restricted to the 1-dof case, but the obtained results can be extended in a straightforward way to multidof manipulators using the VSA-II (or other variable stiffness antagonistic devices). This is sketched in Sect. VI.

\section{Dynamic modeling of The VSA-II}

We recall here the dynamic model of the variable stiffness actuator VSA-II introduced in [10]. The notation is slightly modified, and is similar to the one commonly used for elastic joints of constant stiffness (see, e.g., [26]). This allows also a simpler generalization of the following results to the multidof case (see Sect. VI). The VSA-II actuator is based on a bidirectional antagonistic arrangement of two motors driving a single joint through a flexible transmission system (see Fig. 1). The basic element of the transmission is a 4-bar mechanism. a so-called Grashof neutral linkage, with a linear spring (see Fig. 2), which introduces a nonlinear torquedisplacement characteristic between the input torque applied by the motor and the angular deflection of the joint shaft. We note that the nonlinearity is due to the geometry, not to the spring itself which behaves in the elastic domain. The design is more robust than the original VSA-I prototype [9]. The current version is shown in Fig. 3.

With reference to the left side of Fig. 2, let $\phi$ be the angle connected to the motor position (input) and $\beta$ the angle
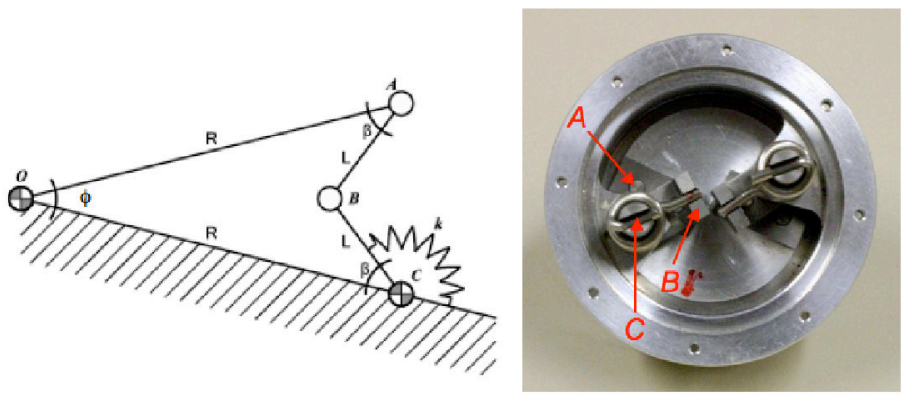

Fig. 2. Left: A single four-bar linkage of the VSA-II (modified from [10]) Right: The assembly of two such linkages for one of the two motors ( $A$, $B$, and $C$ are corresponding points in the two pictures)

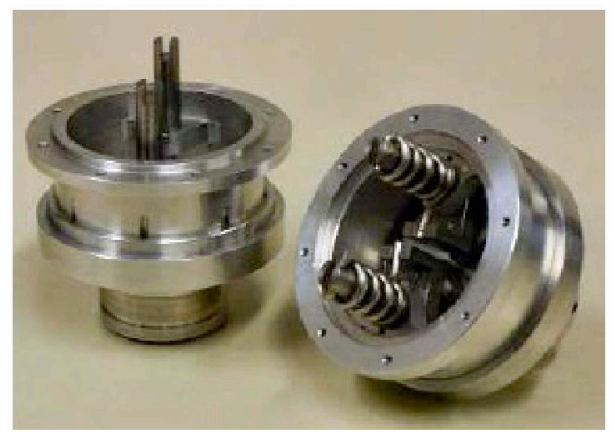

Fig. 3. An open view of the VSA-II prototype (from [10]); there is one such device for each motor on the two sides of the driven joint

connected to the load (output). It is

$$
\beta(\phi)=\arcsin \left(\frac{R}{L} \sin \left(\frac{\phi}{2}\right)\right)-\frac{\phi}{2} .
$$

A mechanical stop ensures the avoidance of the linkage singularity at $\phi=0$. Thus, from the geometry $\phi \in\left(0, \phi_{\max }\right)$, with $\phi_{\max }=2 \arcsin (L / R)$ and $L<R$. The torsional spring of (constant) stiffness $k$ is at rest when $\phi=\beta=0$. Its potential energy is thus $P(\phi)=\frac{1}{2} k \beta^{2}(\phi)$ and the torque at the motor end $O$ due to the deflection $\beta$ is

$$
T(\phi)=\frac{\partial P(\phi)}{\partial \phi}=k \beta(\phi) \frac{\partial \beta(\phi)}{\partial \phi} \geq 0 .
$$

Therefore, the (nonlinear) stiffness seen at $O$ is

$$
\sigma(\phi)=\frac{\partial T(\phi)}{\partial \phi}=k\left(\left(\frac{\partial \beta(\phi)}{\partial \phi}\right)^{2}+\beta(\phi) \frac{\partial^{2} \beta(\phi)}{\partial \phi^{2}}\right)>\sigma_{\mathrm{inf}},
$$

with $\sigma_{\text {inf }}=0.25 k((R / L)-1)^{2}>0$. While the explicit expressions of $T$ and $\sigma$ in (2) and (3) can be found in [10], the above compact forms will be useful for control implementation. The following developments hold true for any other specific form of the nonlinear geometry $\beta(\phi)$ in (1).

In the VSA-II, two such pairs of 4-bar mechanisms are combined for each motor and the two motors are assembled in antagonistic mode as in Fig. 1. Let $\theta_{1}$ and $\theta_{2}$ be the motor positions, and $q$ be the position of the driven link (output load). Replacing $\phi$ in eq. (2) by $\theta_{1}-q$ and $\theta_{2}-q$, respectively for the motor 1 and 2 at the two sides of the joint, the torque 
exerted on the load due to the transmission deflections is given by

$$
\tau_{J}=2\left(T_{1}\left(\theta_{1}-q\right)+T_{2}\left(\theta_{2}-q\right)\right)=2\left(\tau_{J 1}+\tau_{J 2}\right) .
$$

The associated (total) stiffness is ${ }^{1}$

$$
\sigma=\frac{\partial \tau_{J}}{\partial q}=-2\left(\sigma_{1}\left(\theta_{1}-q\right)+\sigma_{2}\left(\theta_{2}-q\right)\right)
$$

where the expression of the functions $\sigma_{i}, i=1,2$, is given by eq. (3).

Consider a 1-dof arm (single link) driven by the VSAII, moving in the vertical plane, and possibly undergoing collisions. The dynamic model can be written as

$$
\begin{aligned}
B \ddot{\theta}_{1}+D \dot{\theta}_{1}+2 \tau_{J 1} & =\tau_{1} \\
B \ddot{\theta}_{2}+D \dot{\theta}_{2}+2 \tau_{J 2} & =\tau_{2} \\
M \ddot{q}+D_{q} \dot{q}+m g d \sin q & =2\left(\tau_{J 1}+\tau_{J 2}\right)+\tau_{K},
\end{aligned}
$$

where $B$ and $M$ are the inertias of the rotor of the two (identical) DC motors and, respectively, of the link (at the joint axis), $D$ and $D_{q}$ are the (small) coefficients of viscous friction at the motor sides and at the link side, $m$ and $d$ are the mass of the link and the distance of its center of mass from the joint, $g$ is the gravity acceleration, $\tau_{1}$ and $\tau_{2}$ are the control torques produced by the two motors, and $\tau_{K}$ is the torque resulting from a link collision (when present).

Based on the model (6), in the next two sections we will first present a nonlinear control design for tracking desired trajectories of the link position $q$ and of the device stiffness $\sigma$, as given by (5), in free motion tasks (i.e., when $\tau_{K}=0$ ), and then introduce a detection scheme and a reaction strategy for handling collisions during motion.

\section{FEEDBACK LINEARIZATION CONTROL}

In [10], a simple linear law of the PD type has been used for controlling both the link motion and the device stiffness. Good results were obtained by suitably combining the control inputs and by defining as controlled outputs the average and the difference of the two motor positions, which are associated respectively to the link position and to the device stiffness. However, this association holds true only at steady-state and in the absence of gravity. A more formal approach is pursued here based on system inversion (or feedback linearization) and considering directly the output of interest, i.e.,

$$
\boldsymbol{y}=\left(\begin{array}{l}
y_{1} \\
y_{2}
\end{array}\right)=\left(\begin{array}{l}
q \\
\sigma
\end{array}\right)
$$

under the action of the control input $\boldsymbol{\tau}=\left(\tau_{1}, \tau_{2}\right)$. The actual computation of a feedback linearization control law depends on the mechanical implementation of the variable stiffness actuation principle. In particular, its applicability relies on the nonsingularity of the decoupling matrix of the system. We will see that the model (6) of a 1-dof arm driven by the VSA-II can be transformed into decoupled chains of

\footnotetext{
${ }^{1}$ The minus sign is due to the chain rule of differentiation, being $\partial \phi / \partial q=-1$. Note that $\sigma$ is always positive.
}

input-output integrators (thus, a linear and easily controllable system), provided that the device is pre-loaded to a positive (and typically, moderate) value of stiffness. As a result of the achieved decoupling, each output component in (7) can be independently and simultaneously controlled.

The system inversion algorithm formally proceeds on the model by differentiating each output component a finite number of times until at least one of the inputs appear. At this differential level, one can try to invert the system and find the expression of the required input-output decoupling control law. If the sum of the orders of output differentiation is equal to the dimension of the state space, then the same decoupling control law achieves also full linearization of the original system, which is what happens here. It should be stressed that no differentiation of measured quantities is actually needed in this control law if the full system state is available. In the following, we assume $\tau_{K}=0$ in (6).

For the first output, we have

$$
\begin{aligned}
y_{1} & =q \\
\dot{y}_{1} & =\dot{q} \\
\ddot{y}_{1} & =\ddot{q}=\frac{1}{M}\left(\tau_{J}-D_{q} \dot{q}-m g d \sin q\right) \\
y_{1}^{[3]} & =\frac{d^{3} q}{d t^{3}}=\frac{1}{M}\left(2\left(\sigma_{1} \dot{\theta}_{1}+\sigma_{2} \dot{\theta}_{2}\right)+\sigma \dot{q}\right. \\
& \left.\quad-D_{q} \ddot{q}-m g d \cos q \dot{q}\right) \\
y_{1}^{[4]} & =b_{1}(\boldsymbol{x})+\frac{2}{M B}\left(\sigma_{1} \tau_{1}+\sigma_{2} \tau_{2}\right),
\end{aligned}
$$

where $b_{1}$ is a function of the state $\boldsymbol{x}=\left(\theta_{1}, \theta_{2}, q, \dot{\theta}_{1}, \dot{\theta}_{2}, \dot{q}\right)$.

In the same way, for the second output

$$
\begin{aligned}
& y_{2}=\sigma \\
& \dot{y}_{2}=\dot{\sigma}=-2\left(\frac{\partial \sigma_{1}}{\partial \theta_{1}} \dot{\theta}_{1}+\frac{\partial \sigma_{2}}{\partial \theta_{2}} \dot{\theta}_{2}\right)+\frac{\partial \sigma}{\partial q} \dot{q} \\
& \ddot{y}_{2}=b_{2}(\boldsymbol{x})-\frac{2}{B}\left(\frac{\partial \sigma_{1}}{\partial \theta_{1}} \tau_{1}+\frac{\partial \sigma_{2}}{\partial \theta_{2}} \tau_{2}\right),
\end{aligned}
$$

with similar functional dependence for $b_{2}$. We can thus write

$$
\left(\begin{array}{c}
y_{1}^{[4]} \\
\ddot{y}_{2}
\end{array}\right)=\boldsymbol{b}(\boldsymbol{x})+\mathcal{A}(\boldsymbol{x})\left(\begin{array}{c}
\tau_{1} \\
\tau_{2}
\end{array}\right),
$$

where the decoupling matrix

$$
\mathcal{A}(\boldsymbol{x})=\boldsymbol{\Gamma}\left(\begin{array}{cc}
\sigma_{1} & \sigma_{2} \\
\frac{\partial \sigma_{1}}{\partial \theta_{1}} & \frac{\partial \sigma_{2}}{\partial \theta_{2}}
\end{array}\right)
$$

is actually only a function of the differences $\theta_{1}-q$ and $\theta_{2}-q$ ( $\boldsymbol{\Gamma}$ is a constant diagonal, and invertible matrix). The determinant of $\mathcal{A}$, evaluated for the mechanical parameters of the VSA-II device, is shown in Fig. 4 as a function of its arguments. One can immediately see that matrix $\mathcal{A}$ is always nonsingular unless $\theta_{1}=\theta_{2}$. Thus, if the VSA-II is kept always in an operation mode where $\theta_{1} \neq \theta_{2}$, the following control law

$$
\left(\begin{array}{l}
\tau_{1} \\
\tau_{2}
\end{array}\right)=\mathcal{A}^{-1}(\boldsymbol{x})\left(\left(\begin{array}{l}
v_{1} \\
v_{2}
\end{array}\right)-\boldsymbol{b}(\boldsymbol{x})\right)
$$



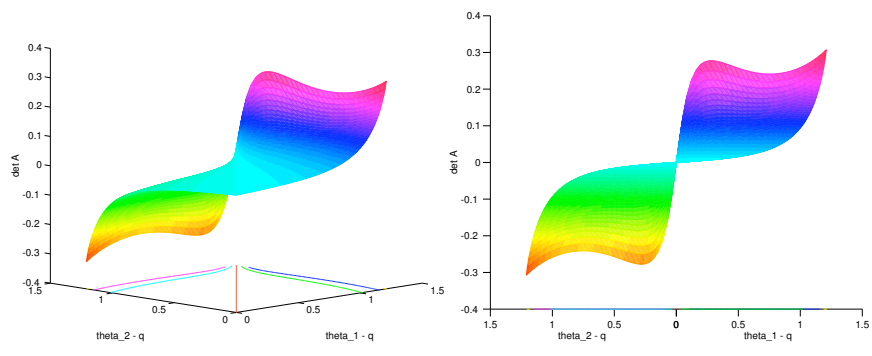

Fig. 4. The determinant of the decoupling matrix $\mathcal{A}$ as a function of its arguments $\theta_{1}-q$ and $\theta_{2}-q$ : $3 \mathrm{D}$ view (left) and side view (right)

solves the decoupling problem, leading to a chain of four integrators between the new control input $v_{1}$ and the output $y_{1}=q$ and a chain of two integrators between the new control input $v_{2}$ and the output $y_{2}=\sigma$. Since the sum $4+2=6=n$, being $n$ the dimension of the system state, the nonlinear state feedback law (12) fully linearizes the closedloop dynamics. As for the computational complexity, we note that the control expression (12) involves only the first four analytic derivatives of the nonlinear function $\beta$ given in (1). Also, gravity compensation is already embedded in the feedback linearizing controller.

In order to guarantee that the condition $\theta_{1} \neq \theta_{2}$ holds at all times, and thus the validity of the proposed control approach, it is sufficient to pre-load the VSA-II at time $t=0$ so that $\theta_{1}(0)-q(0) \neq \theta_{2}(0)-q(0)$, for any initial value $q(0)$ of the link position. Accordingly, the device will display an initial stiffness $\sigma(0)>0$. Imposing now, for an arbitrary motion of $q(t)$, a desired evolution of the stiffness $\sigma_{d}(t)$, with $\sigma_{d}(0)=\sigma(0)$, which is sufficiently bounded away from zero will thus preserve the nonsingularity of matrix $\mathcal{A}$. In fact, this desired behavior $\sigma_{d}(t)$ can be perfectly reproduced thanks to the decoupling and output inversion properties of the control law (12). For this, it will be sufficient to set $v_{2}(t)=\ddot{\sigma}_{d}(t)$, while $v_{1}(t)$ independently defines the evolution of $q(t)$.

More in general, the control design should be completed by specifying linear control laws for $v_{1}$ and $v_{2}$ that stabilize the system to the desired task, expressed in term of link and stiffness behavior. In order to asymptotically reproduce a desired trajectory $q_{d}(t)$ for the link position and, simultaneously, a trajectory $\sigma_{d}(t)$ for the stiffness, we set

$$
\begin{gathered}
v_{1}=q_{d}^{[4]}+k_{q, 3}\left(q_{d}^{[3]}-q^{[3]}\right)+k_{q, 2}\left(\ddot{q}_{d}-\ddot{q}\right) \\
+k_{q, 1}\left(\dot{q}_{d}-\dot{q}\right)+k_{q, 0}\left(q_{d}-q\right) \\
v_{2}=\ddot{\sigma}_{d}+k_{\sigma, 1}\left(\dot{\sigma}_{d}-\dot{\sigma}\right)+k_{\sigma, 0}\left(\sigma_{d}-\sigma\right),
\end{gathered}
$$

where $k_{\sigma, 1}>0, k_{\sigma, 0}>0$, and the gains $k_{q, i}$ are chosen so that $s^{4}+k_{q, 3} s^{3}+k_{q, 2} s^{2}+k_{q, 1} s+k_{q, 0}$ is a (Hurwitz) polynomial having all roots in the left-hand side of the complex plane. The actual values of the control gains in (13) can be chosen, e.g., by pole placement techniques, yielding exponential convergence of the trajectory tracking errors to zero. The higher-order derivatives of $q$ that appear in (13), in the same way as $\sigma$ and its first derivative, can be directly evaluated as functions of the state of the system by means of eqs. (8-9). Thus, there is never a need to differentiate w.r.t. time the (possibly noisy) measured state variables.

A final remark concerns the requirement of smoothness over time for the desired motion and stiffness trajectories. It is apparent from (13) that the link trajectory $q_{d}(t)$ should be differentiable at least four times, whereas the stiffness trajectory $\sigma_{d}(t)$ at least two times. If this is the case, the control law (12-13) will guarantee perfect reproduction for initially matched state conditions, and only exponential tracking otherwise. If a reference trajectory lacks the required smoothness at some point in time, there will be a transient error which is then recovered with the prescribed dynamics imposed by the stabilizing linear control action.

\section{COLlision DETECTION AND REACTION}

Consider now the possible occurrence of a link collision with a human or an obstacle. In eq. (6), it will be $\tau_{K}(t) \not \equiv 0$ for $t \geq t_{K}$, where $t_{K}$ is the instant of first impact.

We would like to detect collisions without the need of additional sensors beyond the encoders available at the joints, possibly allowing the numerical differentiation of position measurements in order to obtain velocities. As mentioned in the introduction, the momentum-based method obtains such a result in rigid robots [20] and in robots with elastic joints of constant stiffness [22]. In the latter case, with the availability of a joint torque sensor one can directly extend the result from the rigid situation. Since the third equation of model (6) is formally equivalent to the link dynamics of a robot with elastic joints, a first solution would be to design the so-called residual allowing to detect collision based only on this equation and on the properties of the link momentum $p=M \dot{q}$. However, this would need sensors for measuring the two nonlinear transmission torques $\tau_{J 1}$ and $\tau_{J 2}$. A more viable but equivalent solution is proposed next.

Denote the sum of the components of the momentum vector of the robot system by

$$
p_{\text {sum }}=B\left(\dot{\theta}_{1}+\dot{\theta}_{2}\right)+M \dot{q}
$$

and define the following residual:

$$
r=k_{I}\left(p_{\text {sum }}-\int_{0}^{t}\left(r+\tau_{1}+\tau_{2}-\tau_{D}-m g d \sin q\right) d s\right)_{(15)}
$$

where $k_{I}>0$ is a free design parameter, the dissipative terms in the model have been collected in $\tau_{D}=D_{q} \dot{q}+D\left(\dot{\theta}_{1}+\dot{\theta}_{2}\right)$, and $r(0)=0$ for a system initially at rest. Using eq. (6), it is easy to check that the residual $r$ satisfies

$$
\dot{r}=k_{I}\left(\tau_{K}-r\right)
$$

resulting in a fist-order, stable filter of the unknown collision torque $\tau_{K}$. For a large $k_{I}$, the residual $r$ follows closely the time behavior of $\tau_{K}$, and in particular returns to zero when the contact is lost. Indeed, to cope with sensor noise and/or model uncertainties that would otherwise generate false alarms, a compatible value of $k_{I}$ should be used in conjunction with some small positive threshold $r_{\text {coll }}$ : collision will be actually detected only at a time instant $t_{D}>t_{K}$ 
when $|r|>r_{\text {coll }}$. Note finally that the computation of the residual (15) is completely independent from the (possibly time-varying) stiffness of the device and from the torques due to deflection of the transmissions. This is an appealing result from an implementation point of view. Moreover, the collision detector works in the same way no matter how the control torques $\tau_{1}$ and $\tau_{2}$ are generated (which is good, e.g., when switching control laws from free motion to collision reaction).

Next, we propose a reaction strategy to be activated upon recognition of a collision. In the present 1-dof case, the situation is trivial because the link can only reverse motion and go in the opposite direction of the detected contact. Anyway, we provide here a general solution that can be used also for the multi-dof case. A first approach could be to switch the control law and apply maximum torque with the two motors, so as to move away from the impact area as fast as possible. However, control on the device stiffness would be given up in this way and this may have a negative effect on the interaction forces during the short time between the detection instant $t_{D}$ and when the link starts executing the commanded reaction. Therefore, it seems more convenient to keep the same feedback linearizing controller (12) and to change only (part of) the linear design of $\boldsymbol{v}=\left(v_{1}, v_{2}\right)$ in eq. (13). In particular, we set

$$
v_{1}=-k_{q, 3} q^{[3]}-k_{q, 2} \ddot{q}-k_{q, 1} \dot{q}+k_{R} r,
$$

where $k_{R}>0$, and the other coefficients are chosen such that $s^{3}+k_{q, 3} s^{2}+k_{q, 2} s+k_{q, 1}$ is a Hurwitz polynomial. The rationale of this law is to have the link be pushed away by an amplified collision torque $\left(k_{R} r \simeq k_{R} \tau_{K}\right)$, while stabilizing motion to $\dot{q}_{d}=0$. After losing contact, $r$ will rapidly go to zero and the link will be slowed down until reaching a rest position away from the collision area. The rest position will depend on the amplitude of the impact force and on the gains chosen in (17). Moreover, the same structure (13) will be kept for $v_{2}$, but dropping the reference value $\sigma_{d}$ to the lowest feasible level of stiffness. This will guarantee the softest compliant behavior of the device in the post-impact phase. Thanks to the decoupling obtained by the control law, in nominal conditions there will be no cross-effects due to the switching of $v_{1}$ and $v_{2}$. However, when external contact is present ${ }^{2}$ and/or due to uncertainty in the dynamic parameters, a nonlinear and coupled behavior still results, see, e.g., [27], which can be evaluated by simulation.

\section{Simulation Results}

The performance of the feedback linearization/decoupling control of Sect. III and of the detection/reaction method of Sect. IV is illustrated here by means of numerical simulations. The following data of the 1-dof VSA-II arm have been experimentally identified on the prototype and are the same used in [10]: $R / L=14 / 8=1.75$ (4-bar linkages), $k=500 \mathrm{~N} \cdot \mathrm{mm} / \mathrm{rad}$ (spring stiffness); $B=7.3 \mathrm{~kg} \cdot \mathrm{m} \cdot \mathrm{mm}$

\footnotetext{
${ }^{2}$ In fact, the unknown collision torque has been neglected in the control design: a non-zero $\tau_{K}$ will affect in $\ddot{q}$ and higher position derivatives as well as $\ddot{\sigma}$ in eqs. (8-9).
}

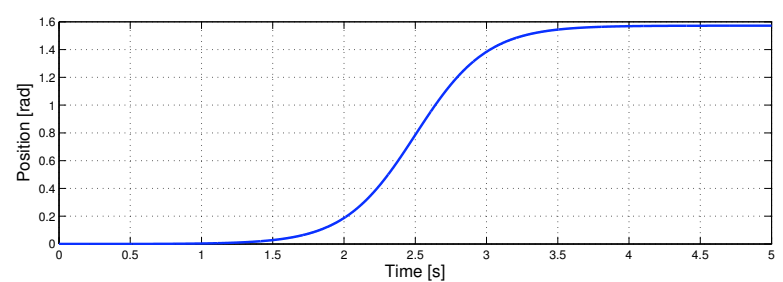

Fig. 5. Evolution of position $q$ (matched initial conditions)

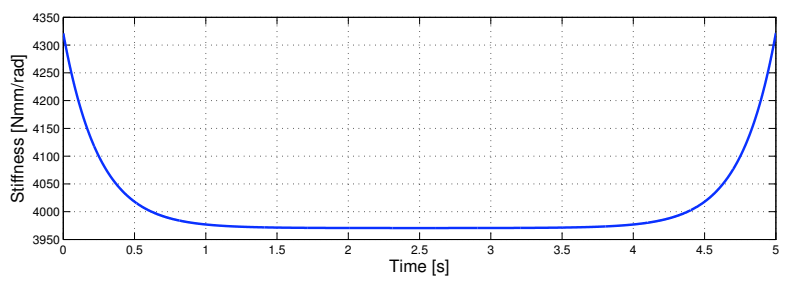

Fig. 6. Evolution of stiffness $\sigma$ (matched initial conditions)

(motor inertia reflected by the reduction ratio); $M=0.1$ $\mathrm{kg} \cdot \mathrm{m} \cdot \mathrm{mm}$ (link inertia at the joint axis, assuming uniform mass); $D=0$ (negligible viscous friction on the motor side); $D_{q}=100 \mathrm{~N} \cdot \mathrm{mm} \cdot \mathrm{s} / \mathrm{rad}$.

\section{A. Free motion}

In the absence of collisions, the reference trajectories for the position and stiffness on a finite time interval $\left[0, T_{\text {tot }}\right]$ are chosen as follows:

$$
\begin{aligned}
& q_{d}(t)=\frac{1}{2 \pi} \frac{1}{1+e^{10-4 t}}+q_{\text {init }} \\
& \sigma_{d}(t)=\frac{1}{\dot{q}_{d}}+\sigma_{\text {init }} .
\end{aligned}
$$

The link should execute an (approximate) rest-to-rest motion of $90^{\circ}$ in $T_{\text {tot }}=5 \mathrm{~s} \mathrm{(} q_{\text {init }}$ sets the link position start at the

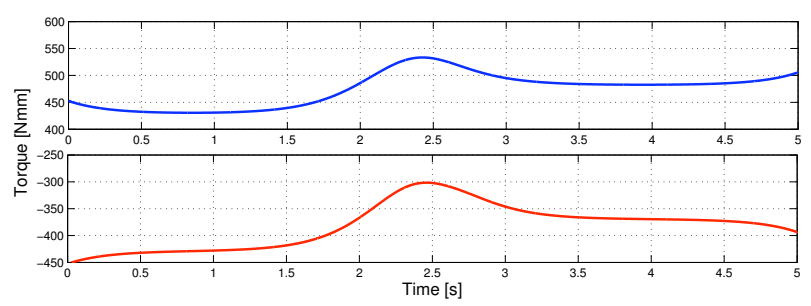

Fig. 7. Control torques $\tau_{1}$ and $\tau_{2}$ (matched initial conditions)

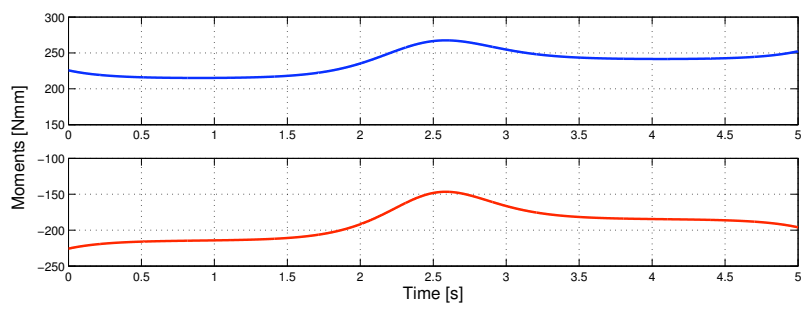

Fig. 8. Deflection torques $\tau_{J 1}$ and $\tau_{J 2}$ (matched initial conditions) 


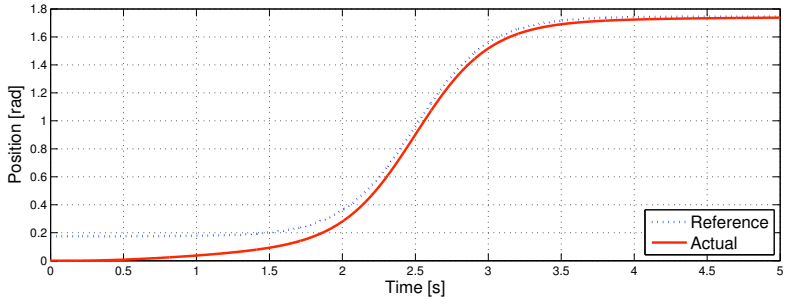

Fig. 9. Evolution of position $q$ for an initial error of $10^{\circ} \approx 0.2 \mathrm{rad}$

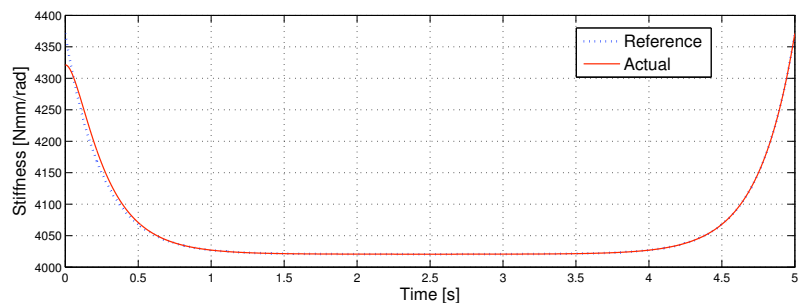

Fig. 10. Evolution of stiffness $\sigma$ for an initial error of $50 \mathrm{~N} \cdot \mathrm{mm} / \mathrm{rad}$

downward equilibrium $q=0$ ), while the stiffness profile complies with the safety rule "stiff when slow, soft when fast" ( $\sigma_{\text {init }}$ sets the desired stiffness at $\left.t=0\right)$. These reference behaviors are reminiscent of the safe brachistochrone proposed in [2], but with conveniently added smoothness.

Figures 5-8 show the results obtained when the initial state of the system is matched with the reference trajectories and their derivatives. Since the state error is initially zero, this condition is kept throughout the motion/stiffness task, independently from the chosen control gains. Moreover, the imposed initial value for stiffness $\sigma$ implies $\theta_{1}(0) \neq \theta_{2}(0)$. Since the control torques remain always bounded, this condition is kept during the whole motion (thus, the decoupling matrix is never singular).

As a second example, the system is started with an initial position error of $10^{\circ}$ and a stiffness error of $50 \mathrm{~N} \cdot \mathrm{mm} / \mathrm{rad}$ with respect to the desired trajectories (18). The control gains in the linear stabilizer (13) were chosen so as to yield two pairs of real poles in -1 and -10 for the position loop, and a double real pole in -10 for the stiffness loop. After a short transient, both position and stiffness recover the desired trajectory (Figs. 9 and 10) without a significant additional control effort (not shown).

\section{B. Collision and reaction}

Next, the presence of a fixed compliant obstacle is considered, located approximately midway along the motion trajectory. Collision occurs around $t_{K}=2.6 \mathrm{~s}$. The results obtained with a detection gain $k_{I}=10^{3}$, a reaction gain $k_{R}=2 \cdot 10^{5}$, and the other gains in eq. (17) chosen so as to have three real poles placed in $\{-110,-87,-23\}$, are shown in Figs. 11-14. After the impact, the reaction strategy moves the link back by about $8^{\circ}$ (this displacement would be larger for smaller gains), while the desired stiffness is controlled so as to drop down to $2000 \mathrm{~N} \cdot \mathrm{mm} / \mathrm{rad}$ (Fig. 12). The residual in Fig. 13, computed from eq. (15), closely

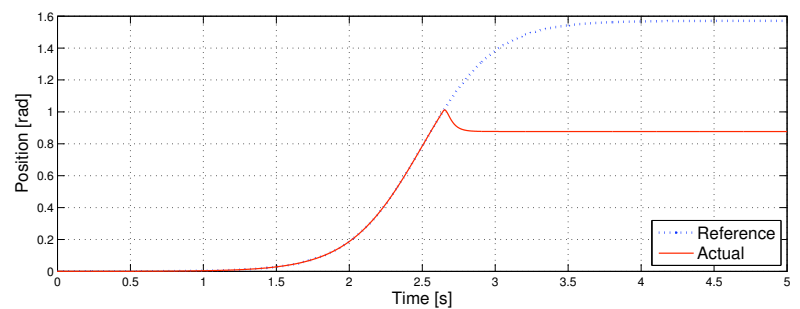

Fig. 11. Evolution of position $q$ in case of collision and reaction

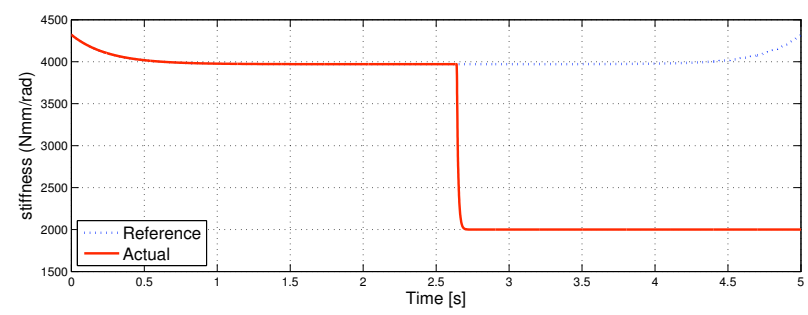

Fig. 12. Evolution of stiffness $\sigma$ in case of collision and reaction

follows the (unmeasured) joint torque resulting from link collision. The control switching is response to the detected collision is evident on the applied torques, which are driven in fact during the reaction phase by the value of $r$ through eq. (17). Note that the steady-state torques in Fig. 14 are those needed to hold the link under gravity in the final rest position: these gravity torques are automatically provided by the feedback linearizing law.

\section{Perturbed conditions}

We have considered the effects of several model perturbations. The controller performance is quite robust with respect to uncertainties up to $30 \%$ of the nominal link and motor inertias $M$ and $B$ alone, with very small tracking
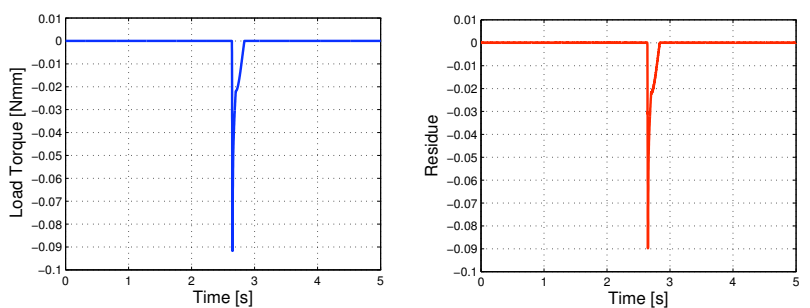

Fig. 13. Evolution of the collision torque $\tau_{K}$ (left) and of the residual $r$ (right): the robot reaction reduces to zero both quantities

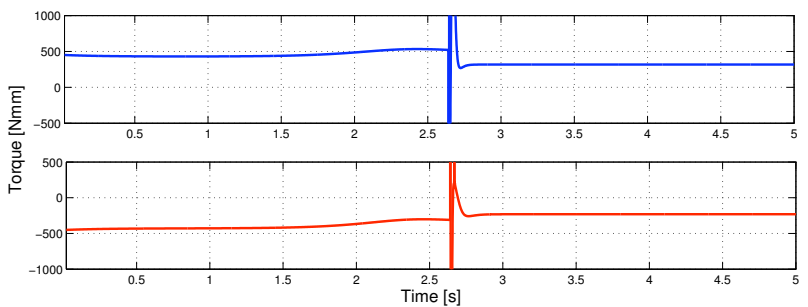

Fig. 14. Control torques $\tau_{1}$ and $\tau_{2}$ in case of collision and reaction 


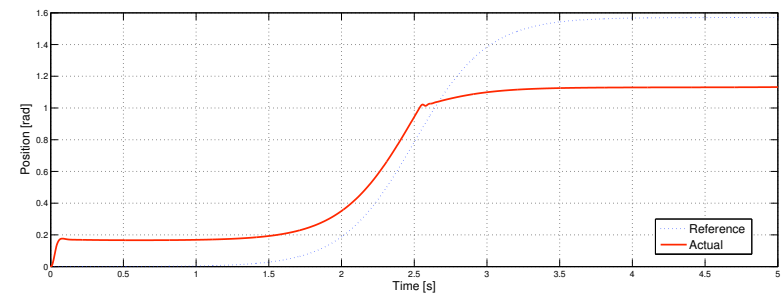

Fig. 15. Perturbed conditions: Position $q$ in case of collision and no reaction

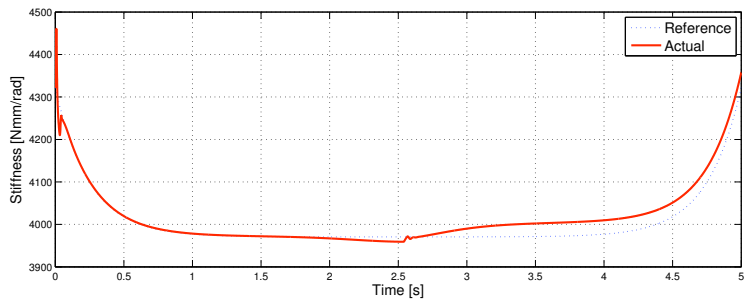

Fig. 16. Perturbed conditions: Stiffness $\sigma$ in case of collision and no reaction

errors. The sensitivity with respect to changes of the spring stiffness $k$ is instead higher, but stability was always preserved for reasonable variations. Figures 15-17 illustrate a free motion/collision situation with model inertias increased by $30 \%$, and an opposite perturbation of + and $-5 \%$ of the spring stiffness on the two motor sides of the VSAII. No collision reaction was activated to better show the effects. During free motion, model uncertainties induce a link position error. This could be reduced by modifying in a robust way the design of the linear controller (13), e.g., by introducing an integral action on the position error channel. After collision, the link bounces slightly against the obstacle and then stops by remaining in contact (compare Fig. 15 with Fig. 11). On the other hand, after an initial transient due to the parameter mismatch, the desired stiffness profile is tracked with sufficient accuracy even after the collision occurrence, as a result of the (now approximate) decoupling with the motion channel (Fig. 16). The evolution of the residual, which is now conveniently low-pass filtered, shows that collision can still be clearly detected, although a higher detection threshold should be used in this case to avoid false alarms due to the model uncertainties (Fig. 17). The final value of $r$ at $t=5 \mathrm{~s}$ is equal to the external contact torque $\tau_{K}$ at steady state.

\section{EXTENSION TO THE MULTI-DOF CASE}

The theoretical developments presented for a 1-dof arm actuated by the VSA-II can be easily extended to the case of N-dof manipulators having each rotational joint equipped with a VSA-II device. In this section, a couple of representative results are sketched.

Under the same assumption used in [15] for modeling robots with elastic joints of constant stiffness, i.e., that the rotational kinetic energy of the rotors of the two motors at each joint is due only to their own spinning, the dynamic

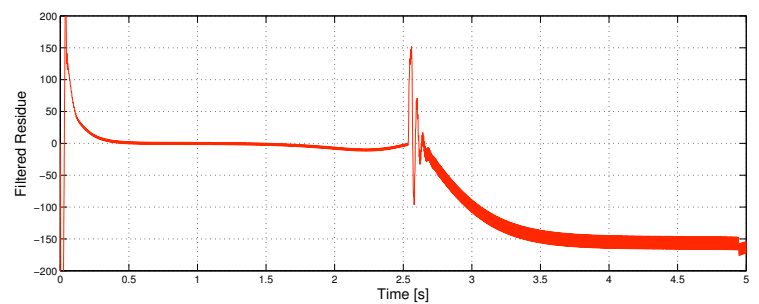

Fig. 17. Perturbed conditions: Residual $r$, low-pass filtered with cut-off at $50 \mathrm{~Hz}$

model (6) can be generalized to the form

$$
\begin{aligned}
\boldsymbol{B} \ddot{\boldsymbol{\theta}}_{1}+\boldsymbol{D} \dot{\boldsymbol{\theta}}_{1}+2 \boldsymbol{\tau}_{J 1} & =\boldsymbol{\tau}_{1} \\
\boldsymbol{B} \ddot{\boldsymbol{\theta}}_{2}+\boldsymbol{D} \dot{\boldsymbol{\theta}}_{2}+2 \boldsymbol{\tau}_{J 2} & =\boldsymbol{\tau}_{2} \\
\boldsymbol{M}(\boldsymbol{q}) \ddot{\boldsymbol{q}}+\boldsymbol{C}(\boldsymbol{q}, \dot{\boldsymbol{q}}) \dot{\boldsymbol{q}}+\boldsymbol{g}(\boldsymbol{q}) & =2\left(\boldsymbol{\tau}_{J 1}+\boldsymbol{\tau}_{J 1}\right)+\boldsymbol{\tau}_{K},
\end{aligned}
$$

where $\boldsymbol{q} \in \mathbb{R}^{N}, \boldsymbol{M}$ is link inertia matrix, $\boldsymbol{C}$ is the factorization matrix of the Coriolis and centrifugal terms, $\boldsymbol{g}$ is the gravity vector term, and $\tau_{K}$ are the joint torques due to collision. Using the same notation of Sect. II, the quantities associated to the variable stiffness joints are $\boldsymbol{B}=\operatorname{diag}\left\{B_{j}\right\}$, $\boldsymbol{D}=\operatorname{diag}\left\{D_{j}\right\}$, and the four $N$-dimensional vectors

$$
\begin{aligned}
& \boldsymbol{\theta}_{i}=\left(\begin{array}{llll}
\theta_{i, 1} & \theta_{i, 2} & \ldots & \theta_{i, N}
\end{array}\right)^{T}, \quad i=1,2, \\
& \boldsymbol{\tau}_{J i}=\left(\begin{array}{llll}
\tau_{J i, 1} & \tau_{J i, 2} & \ldots & \tau_{J i, N}
\end{array}\right)^{T}, \quad i=1,2,
\end{aligned}
$$

with $\tau_{J 1, j}$ and $\tau_{J 2, j}$ for joint $j$ defined as in (4).

The dynamic model (19) can be globally feedback linearized provided that all VSA-II actuators are pre-loaded as in Sect. III, with linearizing coordinates given by $\boldsymbol{q}, \dot{\boldsymbol{q}}, \ddot{\boldsymbol{q}}$, and $\boldsymbol{q}^{[3]}$, together with $\boldsymbol{\sigma}=\left(\begin{array}{ccc}\sigma_{1} & \ldots & \sigma_{N}\end{array}\right)^{T}$ (with $\sigma_{i}$ in the form (5)) and $\dot{\sigma}$. Moreover, collision detection can be achieved using the sum of the generalized momentum vectors pertaining to the motors and to the robot links,

$$
\boldsymbol{p}_{\text {sum }}=\boldsymbol{B}\left(\dot{\boldsymbol{\theta}}_{1}+\dot{\boldsymbol{\theta}}_{2}\right)+\boldsymbol{M}(\boldsymbol{q}) \dot{\boldsymbol{q}},
$$

and defining the residual vector $\boldsymbol{r}$ as

$$
\begin{aligned}
\boldsymbol{r}=\boldsymbol{K}_{I}\left(\boldsymbol{p}_{\text {sum }}-\int_{0}^{t}\right. & \left(\boldsymbol{r}+\boldsymbol{C}^{T}(\boldsymbol{q}, \dot{\boldsymbol{q}}) \dot{\boldsymbol{q}}-\boldsymbol{g}(\boldsymbol{q})\right. \\
+ & \left.\left.\boldsymbol{\tau}_{1}+\boldsymbol{\tau}_{2}-\boldsymbol{D}\left(\dot{\boldsymbol{\theta}}_{1}+\dot{\boldsymbol{\theta}}_{2}\right)\right) d s\right),
\end{aligned}
$$

with $\boldsymbol{K}_{I}>\mathbf{0}$. Using the dynamic properties of the Lagrangian dynamics [22], it can be shown that

$$
\dot{\boldsymbol{r}}=\boldsymbol{K}_{I}\left(\boldsymbol{\tau}_{K}-\boldsymbol{r}\right) .
$$

As in the scalar case, the residual (21) does not require any knowledge about the flexibility (stiffness and deflection torques) of the joints.

\section{CONCLUSIONS}

With reference to the variable stiffness actuation device VSA-II, a complete control strategy has been presented addressing the relevant issues of safety and performance in pHRI.

Using a feedback linearization approach, the actuation capabilities of the device can be fully exploited, under a 
mild condition on stiffness pre-loading, which is sufficient to avoid control singularities. The nonlinear controller is able to track precisely and in a stable way fast trajectories, while imposing independently and with similar accuracy a desired stiffness profile on the fly (performance issue).

A momentum-based observer has been designed so as to promptly detect unexpected collisions without extra joint/transmission torque sensors and independently from the current stiffness value of the device. Based on the residual signal, and taking advantage of the decoupling control in operation, a collision reaction law has been proposed that drives the arm away from the contact area and safely stops it, while dropping suddenly the stiffness to the lowest feasible level. This is useful for minimizing the effects of interaction forces in the early post-impact phase (safety issue).

The combined control strategy proposed in the 1-dof case can be easily extended to articulated manipulators equipped with VSA-II, or other similar variable stiffness actuators of the antagonistic type. In the multi-dof case, the directional information on collision torques embedded in the residual vector is even more relevant for a safe reaction strategy.

We have started the experimentation on the VSA-II prototype to verify also the practical robustness of the approach under real conditions. Future work will address the same control problem for non-antagonistic types of variable stiffness joints, in particular those developed within the European research project PHRIENDS, and will consider alternative collision reaction schemes defined directly at the control torque level.

\section{ACKNOWLEDGEMENTS}

This work has been funded by the European Commission's Sixth Framework Programme as part of the project PHRIENDS under grant no. 045359, and by the MIUR project PRIN 2007 SICURA.

\section{REFERENCES}

[1] A. Bicchi, S. Lodi Rizzini, and G. Tonietti, "Compliant design for intrinsic safety: General issue and preliminary design", in Proc. IEEE/RSJ Int. Conf. on Intelligent Robots and Systems, 2001, pp. 1864-1869.

[2] A. Bicchi and G. Tonietti, "Fast and soft arm tactics: Dealing with the safety-performance trade-off in robot arms design and control", IEEE Robotics and Automation Mag., vol. 11, no. 2, pp. 22-33, 2004.

[3] G. Hirzinger, A. Albu-Schäffer, M. Hähnle, I. Schaefer, and N. Sporer, "On a new generation of torque controlled light-weight robots", in Proc. IEEE Int. Conf. on Robotics and Automation, 2001, pp. 33563363.

[4] K. Ikuta, H. Ishii, and M. Nokata, "Safety evaluation method of design and control for human-care robots", Int. J. of Robotics Research, vol. 22, no. 7/8, pp. 281-297, 2003.

[5] K. A. Wyrobek, E. H. Berger, H. F. M. Van der Loos, and J. K. Salisbury, "Toward a personal robotics development platform: Rationale and design of an intrinsically safe personal robot", in Proc. IEEE Int. Conf. on Robotics and Automation, 2008, pp. 2165-2170.

[6] M. Zinn, O. Khatib, B. Roth, and J. K. Salisbury, "A new actuation approach for human-friendly robot design", Int. J. of Robotics Research, vol. 23, no. 4/5, pp. 379-398, 2005.

[7] J.-J. Park, Y.-J. Lee, J.-B. Song, and H.-S. Kim, "Safe joint mechanism based on nonlinear stiffness for safe human-robot collision", in Proc. IEEE Int. Conf. on Robotics and Automation, 2008, pp. 2177-2182.

[8] S. A. Migliore, E. A. Brown, and S. P. DeWeerth, "Biologically inspired joint stiffness control", in Proc. IEEE Int. Conf. on Robotics and Automation, 2005, pp. 4508-4513.
[9] G. Tonietti, R. Schiavi, and A. Bicchi, "Design and control of a variable stiffness actuator for safe and fast physical human/robot interaction", in Proc. IEEE Int. Conf. on Robotics and Automation, 2005, pp. 528-533.

[10] R. Schiavi, G. Grioli, S. Sen, and A. Bicchi, "VSA-II: A novel prototype of variable stiffness actuator for safe and performing robots interacting with humans", in Proc. IEEE Int. Conf. on Robotics and Automation, 2008, pp. 2171-2176.

[11] J. Choi, S. Park, W. Lee, and S.-C. Kang, "Design of a robot joint with variable stiffness", in Proc. IEEE Int. Conf. on Robotics and Automation, 2008, pp. 1760-1765.

[12] S. Wolf and G. Hirzinger, "A new variable stiffness design: Matching requirements of the next robot generation", in Proc. IEEE Int. Conf. on Robotics and Automation, 2008, pp. 1741-1746.

[13] G. Boccadamo, R. Schiavi, S. Sen, G. Tonietti, and A. Bicchi, "Optimization and fail-safety analysis of antagonistic actuation for pHRI", in European Robotics Symp., Springer Tracts in Advanced Robotics, pp. 109-118. Springer Berlin, 2006.

[14] S. Haddadin, A. Albu-Schäffer, A. De Luca, and G. Hirzinger, "Collision detection and reaction: A contribution to safe physical humanrobot interaction", in Proc. IEEE/RSJ Int. Conf. on Intelligent Robots and Systems, 2008, pp. 3356-3363.

[15] M. W. Spong, "Modeling and control of elastic joint robots", ASME J. of Dynamic Systems, Measurement, and Control, vol. 109, no. 4, pp. 310-319, 1987.

[16] A. De Luca and P. Lucibello, "A general algorithm for dynamic feedback linearization of robots with elastic joints", in Proc. IEEE Int. Conf. on Robotics and Automation, 1998, pp. 504-510.

[17] G. Palli, C. Melchiorri, T. Wimböck, M. Grebenstein, and G. Hirzinger, "Feedback linearization and simultaneous stiffness-position control of robots with antagonistic actuated joints", in Proc. IEEE Int. Conf. on Robotics and Automation, 2007, pp. 4367-4372.

[18] G. Palli, C. Melchiorri, and A. De Luca, "On the feedback linearization of robots with variable joint stiffness", in Proc. IEEE Int. Conf. on Robotics and Automation, 2008, pp. 1753-1759.

[19] S. Morinaga and K. Kosuge, "Collision detection system for manipulator based on adaptive impedance control law", in Proc. IEEE Int. Conf. on Robotics and Automation, 2003, pp. 1080-1085.

[20] A. De Luca and R. Mattone, "Sensorless robot collision detection and hybrid force/motion control", in Proc. IEEE Int. Conf. on Robotics and Automation, 2005, pp. 1011-1016.

[21] A. De Luca and R. Mattone, "Actuator failure detection and isolation using generalized momenta", in Proc. IEEE Int. Conf. on Robotics and Automation, 2003, pp. 634-639.

[22] A. De Luca, A. Albu-Schäffer, S. Haddadin, and G. Hirzinger, "Collision detection and safe reaction with the DLR-III lightweight robot arm", in Proc. IEEE/RSJ Int. Conf. on Intelligent Robots and Systems, 2006, pp. 1623-1630.

[23] K. Suita, Y. Yamada, N. Tsuchida, K. Imai, H. Ikeda, and N. Sugimoto, "A failure-to-safety 'kyozon' system with simple contact detection and stop capabilities for safe human-autonomous robot coexistence", in Proc. IEEE Int. Conf. on Robotics and Automation, 1995, pp. 30893096.

[24] Y. Yamada, Y. Hirasawa, S. Huang, Y. Uematsu, and K. Suita, "Human-robot contact in the safeguarding space", IEEE/ASME Trans. on Mechatronics, vol. 2, no. 4, pp. 230-236, 1997.

[25] J. Heinzmann and A. Zelinsky, "Quantitative safety guarantees for physical human-robot interaction", Int. J. of Robotics Research, vol. 22 , no. $7 / 8$, pp. 479-504, 2003.

[26] A. De Luca and W. Book, "Robots with flexible elements", in Handbook of Robotics, B. Siciliano and O. Khatib, Eds., pp. 287319. Springer, 2008.

[27] R. Ozawa and H. Kobayashi, "Response characteristics of elastic joint robots driven by various types of controllers against external disturbances", in Proc. 6th Int. Conf. on Motion and Vibration Control, 2002, pp. 420-425. 